\title{
COHOMOLOGICAL DIMENSION AND GLOBAL DIMENSION OF ALGEBRAS
}

\author{
JOSEPH A. WEHLEN
}

\begin{abstract}
Let $R$ be a regular local ring and $A$ an algebra over $R$ which is an $R$-progenerator. Defining the cohomological dimension of $A$ as $R-\operatorname{dim} A=1 \cdot h_{A^{*}}(A)$, one obtains the Hochschild cohomological dimension of $A$ as an $R$-algebra. We show the following under the additional hypothesis that $R-\operatorname{dim} A$ is finite: (1) $R-\operatorname{dim} A=n$ iff $A / N$ is $R$-separable and $1 \cdot \mathrm{hd}_{A}(A / N)=n+\mathrm{gl} \operatorname{dim} R$; (2) $\mathrm{gl} \operatorname{dim} A=$ $R$-dim $A+\mathrm{gl} \operatorname{dim} R$; (3) $A$ is $R$-separable iff $\mathrm{gl} \operatorname{dim} A^{e}=\mathrm{gl} \operatorname{dim} R$.
\end{abstract}

The purpose of this note is to extend a result of Eilenberg on the Hochschild cohomological dimension of associative algebras. The results obtained will relate the global dimension of an algebra $A$ which is a progenerator as an $R$-module, the global dimension of the ground ring $R$, and the cohomological dimension of the algebra.

Throughout we will assume that all rings have one. We shall say that an $R$-algebra $A$ is an $R$-progenerator in case $A$ is finitely generated, projective, and faithful as a module over the commutative ring $R$. $N$ will denote the Jacobson radical of the algebra $A$.

We will always mean by "hd" and "gl dim" the left homological dimension and the left global dimension. Recall that if a ring is commutative or noetherian, the left and right global dimensions coincide. We define $R$-dim $A=\operatorname{hd}_{A^{e}}(A)$, where $A^{e}=A \otimes_{R} A^{*}$ and $A^{*}$ is the algebra antiisomorphic to $A$. Since $A^{e *}=A^{e}$, its left and right global dimensions also coincide. Hence for the main theorems, global dimension is well defined.

We require two well-known results, the first of Eilenberg, Rosenberg, and Zelinsky [4, Proposition 2] and the second due to Kaplansky [5, p. 172]:

Result 1. If $A$ and $B$ are $R$-algebras and $A$ is $R$-flat, then (a) $\mathrm{gl} \operatorname{dim} B \otimes A \leqq R-\operatorname{dim} A+\mathrm{gl} \operatorname{dim} B$. If further, $A$ is also $R$-projective and contains $R$ as an $R$-direct summand, then (b) $\operatorname{gl} \operatorname{dim} B \leqq \mathrm{gl} \operatorname{dim} B \otimes A$.

Received by the editors December 21, 1970.

AMS 1970 subject classifications. Primary 18H15, 16A46, 13H05; Secondary 13D05, $16 \mathrm{~A} 62$.

Key words and phrases. Hochschild cohomological dimension, regular local ring, separable algebra, global dimension, progenerator.

(C) American Mathematical Society 1972 
Whenever $A$ is an $R$-progenerator, $R$ is an $R$-direct summand of $A$ and hence Result 1 applies in its entirety.

RESULT 2. Let $(x)$ be an ideal of any ring $R, x$ a central nonzero divisor. If $M$ is an $R /(x)$-module of finite homological dimension over $R /(x)$, then $\operatorname{hd}_{R}(M)=\mathrm{hd}_{R /(x)}(M)+1$.

Combining a result of Auslander [1, Corollary 11] with a result of Eilenberg [3, Corollary to Theorem III], we have the following wellknown

RESUlt 3. If $A$ is a finitely generated algebra over a field $R$, and $R-\operatorname{dim} A$ is finite, then $R-\operatorname{dim} A=n$ iff $A / N$ is $R$-separable and $n=$ $\mathrm{gl} \operatorname{dim} A=\mathrm{hd}_{A}(A / N)$.

Finally, [7, Theorem 2.1] shows

RESULT 4. If $A$ is a finitely generated, projective $R$-algebra and $R$-dim $A$ is finite, $R-\operatorname{dim} A=n$ iff $R / m-\operatorname{dim} A / m A \leqq n$ for all maximal ideals $\boldsymbol{m}$ of $R$ with equality holding for some $\boldsymbol{m}$. If $R$ is a local ring, $A / N$ is separable and $R-\operatorname{dim} A=R / \boldsymbol{a}-\operatorname{dim} A / \boldsymbol{a} A$ for every proper ideal $\boldsymbol{a}$ of $R$.

For the remainder of the paper, we shall assume that an $R$-algebra $A$ is an $R$-progenerator. If $R$ is a local ring, that $A$ is faithful is a consequence of its being finitely generated and projective.

Recall that a regular local ring $R$ is a commutative noetherian ring with a unique maximal ideal with the additional property that $R$ has finite global dimension. Since every regular local ring is an integral domain, every nonzero element of $R$ is a nonzero divisor of $A$ whenever $A$ is $R$-projective.

THEOREM A. Let $R$ be a regular local ring, $A$ an $R$-progenerator of finite cohomological dimension. $R-\operatorname{dim} A=n$ iff $A / N$ is $R$-separable and $\operatorname{hd}_{A}(A / N)=n+\mathrm{gldim} R$.

Proof. We proceed by induction on the $\mathrm{gl} \operatorname{dim} R$. If $\mathrm{gl} \operatorname{dim} R=0$, the theorem reduces to Result 3. Suppose it is true for all $r$ less than $k$. Let $\mathrm{gl} \operatorname{dim} R=k$. Then there is a principal minimal prime $(x)$ such that gl $\operatorname{dim} R /(x)=k-1$ by [6, p. 73]. Now $R-\operatorname{dim} A /(x)=R /(x)-\operatorname{dim} A /(x)=$ $R-\operatorname{dim} A=n$ iff $A / N$ is $R$-separable and $\operatorname{hd}_{A /(x)}(A / N)=\operatorname{gldim} R /(x)+n$ by the inductive hypothesis. Then, by Result 2 , this is true iff $A / N$ is $R$ separable and $\operatorname{hd}_{A}(A / N)=n+\mathrm{gl} \operatorname{dim} R$.

COROLLARY. If we further assume that either $\operatorname{gl} \operatorname{dim} R$ or $R-\operatorname{dim} A$ is positive, $R-\operatorname{dim} A=n$ iff $A / N$ is $R$-separable and $\mathrm{hd}_{A}(N)=\mathrm{gl} \operatorname{dim} R+n-1$.

Proposition 1. $\mathrm{gl} \operatorname{dim} R \leqq \mathrm{gl} \operatorname{dim} A \leqq R-\operatorname{dim} A+\mathrm{gl} \operatorname{dim} R$.

Proof. Apply both parts of Result 1 with $(A, B)$ replaced by $(A, R)$.

Proposition 2. $\mathrm{gl} \operatorname{dim} R \leqq \mathrm{gl} \operatorname{dim} A^{e} \leqq 2 R-\operatorname{dim} A+\mathrm{gl} \operatorname{dim} R$. 
Proof. Apply (a) and (b) with $(A, B)$ replaced by $\left(A^{*}, A\right)$ and $\left(A^{e}, R\right)$ respectively. Note that $R-\operatorname{dim} A^{*}=R-\operatorname{dim} A$ [2, IX, Remark 1, p. 171]. Then apply Proposition 1.

Proposition 3. $\mathrm{gl} \operatorname{dim} R \leqq \mathrm{gl} \operatorname{dim} A \leqq \mathrm{gl} \operatorname{dim} A^{e}$.

Proof. Apply (b) twice, with $(A, B)$ replaced by $(A, R)$ and then by $\left(A^{*}, A\right)$.

THEOREM B. Let $R$ be a regular local ring, $A$ an $R$-progenerator of finite cohomological dimension

$$
\mathrm{gl} \operatorname{dim} A=R-\operatorname{dim} A+\mathrm{gl} \operatorname{dim} R .
$$

(One should note that this result is already known for semiprimary algebras over a field and for polynomial algebras.)

Proof. Recall that $R-\operatorname{dim} A=\mathrm{gl} \operatorname{dim} A / \boldsymbol{m} A$ since $R$ is local by Results 3 and 4 . We again prove the result by induction on $\operatorname{gl} \operatorname{dim} R$. Let $(x)$ be a principal minimal prime such that $\mathrm{gl} \operatorname{dim} R=\mathrm{gl} \operatorname{dim} R /(x)+1$. Taking suprema over all $A /(x)$-modules in Result 2 together with Proposition 1, we have that $\mathrm{gl} \operatorname{dim} A /(x)+1 \leqq \mathrm{gl} \operatorname{dim} A \leqq R-\operatorname{dim} A+\mathrm{gl} \operatorname{dim} R$. By the inductive hypothesis, $g l \operatorname{dim} A /(x)=R /(x)-\operatorname{dim} A /(x)+\mathrm{gl} \operatorname{dim} R /(x)=$ $R-\operatorname{dim} A+\mathrm{gl} \operatorname{dim} R-1$. Hence it follows that $(\mathrm{gl} \operatorname{dim} A /(x)+1=)$ $\mathrm{gl} \operatorname{dim} A=R-\operatorname{dim} A+\mathrm{gl} \operatorname{dim} R$.

COROLlary B.1. Under the same hypotheses, we have that $\operatorname{gl} \operatorname{dim} A=$ $\mathrm{hd}_{A}(A / N)$.

Recall that a ring is said to be hereditary if every ideal is projective, but the ring is not semisimple (i.e., the global dimension is one).

COROLlary B.2. Under the same hypotheses, we have that $A$ is hereditary iff

(a) $A$ is separable and $R$ is a local PID or

(b) $R-\operatorname{dim} A=1$ and $R$ is a field.

CoRollary B.3. Under the same hypotheses, we have that $N$ is projective as an A-module iff $A$ is $R$-separable.

THEOREM C. Let $R$ be a regular local ring, $A$ an $R$-progenerator of finite cohomological dimension

$$
\mathrm{gl} \operatorname{dim} A^{e}=2 R-\operatorname{dim} A+\mathrm{gl} \operatorname{dim} R .
$$

Proof. By Theorem B, gl $\operatorname{dim} A^{e}=R-\operatorname{dim} A^{e}+\mathrm{gl} \operatorname{dim} R$. Applying Theorem 7.4 [2, IX] together with Result 4, one obtains

$$
\begin{aligned}
R-\operatorname{dim} A^{e} & =R / m-\operatorname{dim}(A / \boldsymbol{m} A)^{e} \\
& =R / \boldsymbol{m}-\operatorname{dim} A / \boldsymbol{m} A+R / m-\operatorname{dim} A^{*} / \boldsymbol{m} A^{*}=2 R-\operatorname{dim} A .
\end{aligned}
$$


(One should notice that it is easy to obtain the inequality gl $\operatorname{dim} R+$ $R$-dim $A \leqq \mathrm{gl} \operatorname{dim} A^{e} \leqq 2 R-\operatorname{dim} A+\mathrm{gl} \operatorname{dim} R$ from Theorem B together with the left-hand inequalities of Propositions 2 and 3.)

Corollary C.1. Under the same hypotheses, $\mathrm{gl} \operatorname{dim} A^{e}=\mathrm{gl} \operatorname{dim} R$ iff $A$ is R-separable.

This corollary is an interesting extension of a theorem contained in Cartan and Eilenberg [2, IX, Theorem 7.9].

We can describe a larger class of rings $R$ and algebras $A$ for which Theorems $\mathrm{B}$ and $\mathrm{C}$ hold in the following way. We shall say that an $R$ algebra $A$ is cohomologically isodimensional if $R_{m}$-dim $A_{m}=R-\operatorname{dim} A$ for every maximal ideal $\boldsymbol{m}$ of $R$. We shall call a commutative ring $R$ globally isodimensional provided $\mathrm{gl} \operatorname{dim} R_{m}=\mathrm{gl} \operatorname{dim} R$ for every maximal ideal $\boldsymbol{m}$ of $R$.

THEOREM D. Let $R$ be any commutative noetherian ring of finite global dimension. Let $A$ be an $R$-progenerator which is an R-algebra of finite cohomological dimension. Assume also that either (a) $R$ is globally isodimensional or (b) $A$ is cohomologically isodimensional. Then the following hold:

(1) $\mathrm{gl} \operatorname{dim} A=R-\operatorname{dim} A+\mathrm{gl} \operatorname{dim} R$.

(2) $\mathrm{gl} \operatorname{dim} A^{e}=2 R-\operatorname{dim} A+\mathrm{gl} \operatorname{dim} R$.

(3) $\mathrm{gl} \operatorname{dim} A^{e}=\mathrm{gl} \operatorname{dim} R$ iff $A$ is $R$-separable.

(4) If $R$ is semilocal, $\operatorname{hd}_{A}(A / N)=R-\operatorname{dim} A+\mathrm{gl} \operatorname{dim} R=\mathrm{gl} \operatorname{dim} A$.

Proof. We shall indicate only the proofs of (1) and (4) in the case where $R$ is globally isodimensional, as the rest follow in a similar way. Recall that for any finitely generated algebra $A$ over a noetherian ring $R$, $\mathrm{gl} \operatorname{dim} A=\sup \left[\mathrm{gl} \operatorname{dim} A_{\boldsymbol{m}}\right]$ and $\mathrm{gl} \operatorname{dim} R=\sup \left[\mathrm{gl} \operatorname{dim} R_{\boldsymbol{m}}\right]$ where $\boldsymbol{m}$ runs through the maximal ideals of $R$.

Let $\boldsymbol{m}$ be a maximal ideal of $R$ such that $R-\operatorname{dim} A=R_{m}-\operatorname{dim} A_{m}$. Then

$$
\begin{aligned}
\mathrm{gl} \operatorname{dim} A_{m} & =R_{m}-\operatorname{dim} A_{m}+\mathrm{gl} \operatorname{dim} R_{m} \\
& =R-\operatorname{dim} A+\mathrm{gl} \operatorname{dim} R
\end{aligned}
$$

by Theorem B. Hence, $\mathrm{gl} \operatorname{dim} A \geqq R-\operatorname{dim} A+\mathrm{gl} \operatorname{dim} R$. Equality follows from Proposition 1.

For (4), let $\boldsymbol{m}$ be one of the maximal ideals of $R$ and let $J(R)$ denote the Jacobson radical of $R$. An application of the Chinese Remainder Theorem gives the exact sequence

$$
0 \rightarrow N \rightarrow A \rightarrow \prod_{i=1}^{n} A_{i} / N_{i} \rightarrow 0
$$

where $n$ is the number of maximal ideals of $R, \prod A_{i}$ is the canonical direct 
product decomposition of $A / J(R) \cdot A$ generated by the idempotents of $R / J(R)$, and $N_{i}$ is the Jacobson radical of $A_{i}$. Result 3 clearly guarantees the separability of $A_{i} / N_{i}$ over $R / m_{i}$.

Since $R_{m}$ is $R$-flat, we obtain the exact sequence

$$
0 \rightarrow N_{m} \rightarrow A_{m} \rightarrow A_{j} / N_{j} \rightarrow 0
$$

where $A_{j}$ is the summand over $R / m$.

Thus it follows that $N_{m}$ is the Jacobson radical of $A_{m}$. Hence Theorem A gives $\mathrm{hd}_{A_{m}}(A / N)_{m}=\mathrm{hd}_{A_{m}}\left(A_{m} / N_{m}\right)=R_{m}$-dim $A_{m}+\mathrm{gldim} R_{m}$. Using the well-known result for noetherian algebras that for $M$ finitely generated $\mathrm{hd}_{A}(M)=\sup \left[\mathrm{hd}_{A_{m}}\left(M_{m}\right)\right]$, the proof now proceeds as in part (1).

Finally, we note the following entertaining

COROLlaRY D.1. Let $A$ be an R-progenerator which is an R-algebra of finite cohomological dimension. Let $R$ be a noetherian ring of finite global. dimension. Any two of the following imply the third:

(a) $A$ is cohomologically isodimensional;

(b) $R$ is globally isodimensional;

(c) $\mathrm{gl} \operatorname{dim} A=\mathrm{gl} \operatorname{dim} A_{m}$ for every maximal ideal $\boldsymbol{m}$ of $R$.

We note that the hypothesis of local or semilocal is necessary for Theorems $\mathrm{A}$ and $\mathrm{D}(4)$ as the $2 \times 2$ upper triangular matrix algebra over the rational integers shows. Although the integers are hereditary, the radical of the algebra is easily seen to be projective as a module over the algebra. On the other hand, the rational integers are globally isodimensional; so the algebra has global dimension 2 .

In fact, it can be shown that

THEOREM E. If $R$ is a dedekind domain with infinitely many primes and $A$ is an $R$-algebra such that $A / N$ is separable and $A$ is an R-progenerator of finite cohomological dimension and if either $R$ is globally isodimensional or $A$ is cohomologically isodimensional, then $R-\operatorname{dim} A=\mathrm{hd}_{A}(A / N)$.

Proof. Since $N$ is nilpotent, one can readily verify that $A / N$ is torsionfree and hence projective. Moreover, the separability of $A / N$ guarantees that $(N+\boldsymbol{m} A) / \boldsymbol{m} A$ is the Jacobson radical of $A / \boldsymbol{m} A$. By Results 3 and 4 and the arguments of [7, Theorem 2.1], we have the following chain of equalities:

$$
\begin{aligned}
R-\operatorname{dim} A & =\sup [R / \boldsymbol{m}-\operatorname{dim} A / \boldsymbol{m} A]=\sup \left[\mathrm{hd}_{A / \boldsymbol{m} A}(A /(N+\boldsymbol{m} A))\right] \\
& =\operatorname{hd}_{A}(A / N),
\end{aligned}
$$

where the supremum is taken over all maximal ideals $\boldsymbol{m}$ of $R$. 
Propositions 2 and 3 make it clear that the global dimension of $R$ must be finite since there exist algebras which are $R$-progenerators of every cohomological dimension over any ring $R$.

It is uncertain what role the hypothesis of noetherian plays, for any local nonnoetherian domain $R$ with a minimal generating set for $\boldsymbol{m}$ consisting of $n$ elements $\left(x_{1}, \cdots, x_{n}\right)$ such that $\left(x_{1}, \cdots, x_{i}\right)$ is a prime ideal for $1 \leqq i \leqq n$ has the properties of Theorem $\mathrm{A}$ through $\mathrm{C}$ provided $\operatorname{gl} \operatorname{dim} R$ is replaced by $n$.

\section{BIBLIOGRAPHY}

1. M. Auslander, On the dimension of modules and algebras. III: Global dimension, Nagoya Math. J. 9 (1955), 67-77. MR 17, 579.

2. H. Cartan and S. Eilenberg, Homological algebra, Princeton Univ. Press, Princeton, N.J., 1956. MR 17, 1040.

3. S. Eilenberg, Algebras of cohomologically finite dimension, Comment. Math. Helv. 28 (1954), 310-319. MR 16, 442.

4. S. Eilenberg, A. Rosenberg and D. Zelinsky, On the dimension of modules and algebras. VIII: Dimension of tensor products, Nagoya Math. J. 12 (1957), 71-93. MR 20 \#5229.

5. I. Kaplansky, Fields and rings, Univ. of Chicago Press, Chicago, Ill., 1969.

6. D. G. Northcott, Ideal theory, Cambridge Tracts in Math. and Math. Phys., no. 42, Cambridge Univ. Press, 1953. MR 15, 390.

7. J. A. Wehlen, Algebras of finite cohomological dimension, Nagoya Math. J. 43 (1971), 127-135.

Department of Mathematics, Ohio University, Athens, Oho 45701 